Portland State University

PDXScholar

Electrical and Computer Engineering Faculty

Publications and Presentations

Electrical and Computer Engineering

2-2015

\title{
Semi-modular Delay Model Revisited in Context of Relative Timing
}

Hoon Park

Portland State University, parkhoon@gmail.com

Anping $\mathrm{He}$

Lanzhou University

Marly Roncken

Portland State University

Xiaoyu Song

Portland State University

Follow this and additional works at: https://pdxscholar.library.pdx.edu/ece_fac

Part of the Computer Engineering Commons, Computer Sciences Commons, and the Electrical and Computer Engineering Commons

Let us know how access to this document benefits you.

\section{Citation Details}

Park, Hoon; He, Anping; Roncken, Marly; and Song, Xiaoyu, "Semi-modular Delay Model Revisited in Context of Relative Timing" (2015). Electrical and Computer Engineering Faculty Publications and Presentations. 307.

https://pdxscholar.library.pdx.edu/ece_fac/307

This Post-Print is brought to you for free and open access. It has been accepted for inclusion in Electrical and Computer Engineering Faculty Publications and Presentations by an authorized administrator of PDXScholar. Please contact us if we can make this document more accessible: pdxscholar@pdx.edu. 


\section{The Semi-Modular Delay Model Revisited in the Context of Relative Timing}

\author{
Hoon Park, Anping He, Marly Roncken, Xiaoyu Song
}

\begin{abstract}
We present a new definition of semi-modularity to accommodate relative timing constraints in self-timed circuits. While previous definitions ignore such constraints, the new definition takes them into account. We illustrate the difference on a design solution for a well-known speedindependent circuit implementation of the Muller C element and a set of relative timing constraints that renders the implementation hazard-free. The old definition produces a false semi-modularity conflict that canno exist due to the set of imposed constraints. The new definition correctly accepts the solution.
\end{abstract}

Introduction: Semi-modularity is a well-known paradigm for designing hazard-free self-timed digital circuits. Relative timing is an alternative paradigm, used for the same purpose. The two paradigms were introduced independently, and for different reasons. We will show that this overlap in purpose unnecessarily limits the set of relative timing constraints.

Semi-modularity requires that a digital signal change - when enabledmust happen before it is disabled. Semi-modularity played a key role in the early development of computer aided design tools for self-timed systems. Introduced by Raymond Miller [1], it was the starting point for the first generation of self-timed design and analysis tools [2, 3, 4]. The early focus was on generating circuits that-though large and slowwere correct, independent of the gate and wire delays in the design.

Armed with theory and tools, the next generation could switch gear Focus shifted to speed and energy efficiency, which were achieved by exchanging delay-insensitivity for extra delay assumptions formulated as relative timing constraints $[5,6,7,8,9]$. But the definitions of semi-modularity were neither re-examined nor adapted in the context of relative timing. In this paper, we will show the need for and present a new definition of semi-modularity that is aware of relative timing constraints.

Semi-modularity—old definition: Semi-modularity expresses that a digital signal change - when enabled - must happen before it is disabled. It's a "no transition left behind" type of paradigm. Transitions in a digital circuit refer to gate or wire transitions. Both types of transitions can be modeled as gate transitions, by adding "dummy" buffer gates in each wire branch [10].

A digital gate can be described as a boolean function, $F$, from its inputs, $i n_{i, i=1 . . n}$, to its outputs. For simplicity and without loss of generality, the focus in this paper is on single-output gates. A gate whose output, out, is consistent with its inputs is said to be stable-its output and next output, $o u t^{\prime}$, have the same value as the function value on the current inputs:

$$
\text { out }=F\left(\text { in }_{1}, \cdots, i n_{n}\right) \wedge \text { out }^{\prime}=\text { out }
$$

A gate whose output is inconsistent with its inputs is said to be unstableits output differs from the function value on its inputs:

$$
\text { out } \neq F\left(i n_{1}, \cdots, i n_{n}\right)
$$

(unstable)

An unstable gate can become stable by changing either its output or its inputs. Semi-modularity allows the output but forbids the input change.

Our execution model for changes is based on finite traces of events, i.e. gate or wire transitions, and on an interleaving semantics that represents parallel events by arbitrary sequential orderings of the events. The resulting single event orderings are also used to model the delays of the events, relative to each other. This is a standard execution model for analyzing self-timed designs. Under this model, semi-modularity for gates with arbitrary transition delays can be formulated as follows:

Definition 1 (semi-modular gate delay model-original version): An unstable gate sees no changes until its output changes, i.e.

$$
\begin{aligned}
& \text { out } \neq F\left(i n_{1}, \cdots, i n_{n}\right) \rightarrow \\
& \left(\left[\text { out }^{\prime}=F\left(\text { in }_{1}, \cdots, \text { in }_{n}\right) \vee \text { out }^{\prime}=\text { out }\right]\right. \\
& \left.\wedge F\left(i n_{1}^{\prime}, \cdots, i n_{n}^{\prime}\right)=F\left(i n_{1}, \cdots, i n_{n}\right)\right)
\end{aligned}
$$

Relative Timing constraints: Only a small class of self-timed circuits can work correctly under arbitrary gate and wire delays. Most of the early circuits had relative timing constraints, though these might have been called "isochronic forks" [11] and gone unidentified because wire delays were often ignored at the time. Relative timing constraints specify event orderings that-when obeyed - guarantee correct operation of the circuit. There are various ways to express these constraints. In this paper, we follow $[8,9]$ and express them as triples (POD, EARLY, LATE), where:

- POD is the "point of divergence" event that causes the target events.

- EARLY is the target event following POD that must happen first.

- LATE is the target event following POD that must happen last.

Relative timing constraints are guaranteed by adjusting the delay settings of gates and wires in the circuit, and validated using static timing analysis.

We can integrate relative timing constraints into our execution model by adjusting the way an unstable gate may change. In particular, we must avoid gate changes that conflict with the relative timing constraints in the circuit. We do this as follows. For every gate output, out, with rising or falling transition out + or out- used as a LATE event of some relative timing triple (POD, EARLY, LATE), we block the gate transition whenever we've seen POD but not yet EARLY. This blocking procedure is easy to implement [9]. We assume that it is available in our execution model as a boolean function, block(.), operating on transitions-e.g. block (out + ) holds if and only if out + is blocked by a constraint. Fig. 1 summarizes which output changes are enabled in the new model, and introduces some graphical notations to help visualize what's going on.

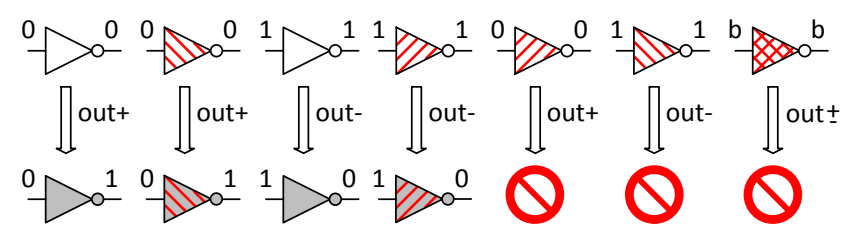

Fig. 1 Summary of enabled and forbidden output transitions in the new execution model, illustrated for an inverter which executes the boolean function out $=\neg i n$. The b's at the top right are both 0 or both 1 . We color the unstable gates white, the stable gates grey. We add red stripes to gates with blocked output transitions: / or \or X indicate that respectively the rising, the falling, or both transitions are blocked. Only the four upper-left gates are enabled to change their output and become the stable lower-left gates.

Semi-modularity—new definition: As illustrated in Fig. 1, a gate output change under the new execution model is enabled if and only if the gate is unstable (for its given inputs and output) and the transition that causes the output change is not blocked by relative timing constraints. As before, semi-modularity requires that a digital signal change-when enabledmust happen before it is disabled. This leads to the following definition of semi-modularity in the new execution model with relative timing:

Definition 2 (semi-modular gate delay model-new version): An unblocked unstable gate sees no changes until its output changes, i.e.

$$
\begin{aligned}
& \left(\text { out } \neq F\left(i n_{1}, \cdots, i n_{n}\right)\right. \\
& \wedge[(\text { out } \wedge \neg \text { block }(\text { out }-)) \vee(\neg \text { out } \wedge \neg \text { block }(\text { out }+))]) \\
& \left(\left[\text { out }^{\prime}=F\left(\text { in }_{1}, \cdots, \text { in }_{n}\right) \vee \text { out }^{\prime}=\text { out }\right]\right. \\
& \left.\wedge F\left(i n_{1}^{\prime}, \cdots, i n_{n}^{\prime}\right)=F\left(i n_{1}, \cdots, i n_{n}\right)\right)
\end{aligned}
$$

Comparison: We will use use the design setup in Fig. 2 to illustrate the different execution semantics for Definitions 1 and 2. Fig. 2 shows a wellknown circuit implementation of the Muller $\mathrm{C}$ element, with its intended behavior specified as a signal transition graph, and four relative timing constraints represented as (POD, EARLY, LATE) triples. The circuit uses two input signals, $A$ and $B$, and one output signal, $C$. Initially, all three signals are 0 : the specification starts in the double circle, and the initial values for internal signals in the circuit are indicated in the picture. We follow the color scheme of Fig. 1. The white (unstable) inverters represent the environment, and the grey (stable) nand gates represent the Muller $\mathrm{C}$ element proper. The circuit is intended to be speed-independent-i.e. wire transitions can be ignored. The circuit gates are addressed by their output signal name. Signals observable to the specification may have two names-e.g. $A$ and $a$ represent the same signal. 

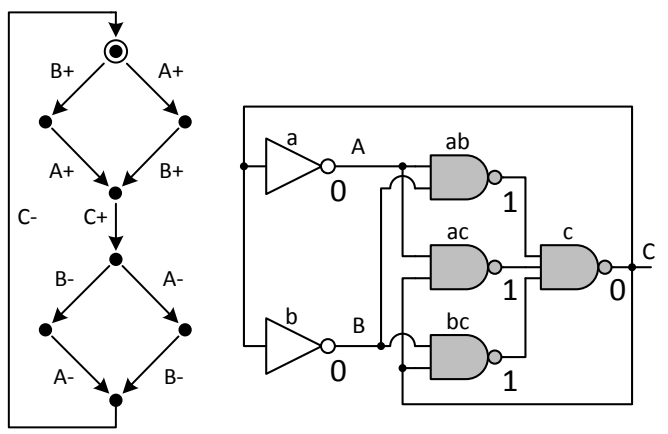

Constraints:

(c+, ac-, a-)

$(\mathrm{c}+, \mathrm{ac}-, \mathrm{c}-)$

$(\mathrm{c}+, \mathrm{bc}-, \mathrm{b}-)$

$(\mathrm{c}+, \mathrm{bc}-, \mathrm{c}-)$

Fig. 2 Design setup for a speed-independent Muller $C$ element, with its specification (left), circuit (middle), and relative timing constraints (right). The inverters execute out $=\neg$ in, the nand gates out $=\neg\left(i n_{1} \wedge \cdots \wedge i n_{n}\right)$.
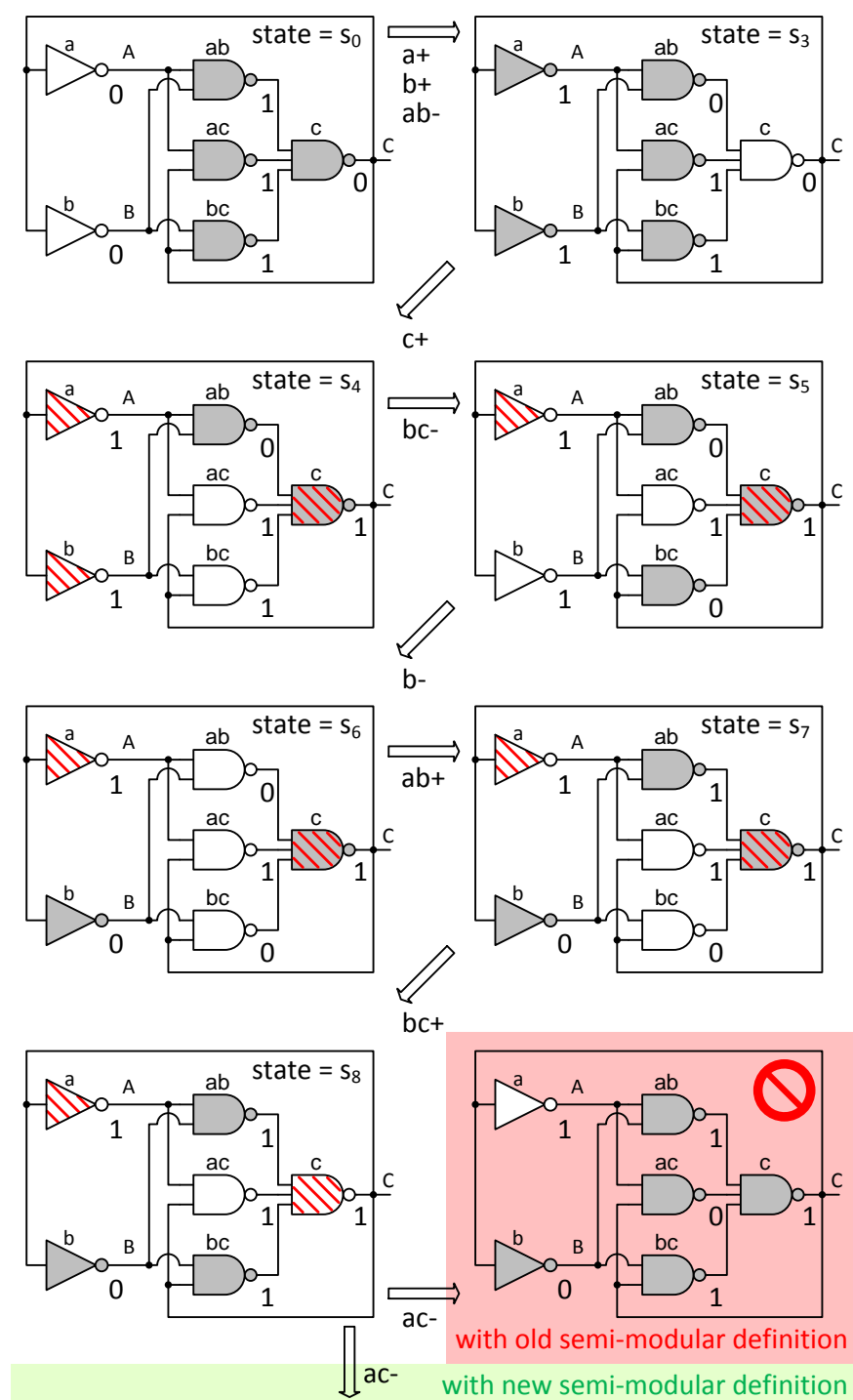

$\mathrm{bc}+$

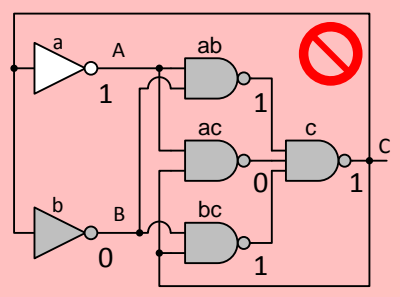

with old semi-modular definition

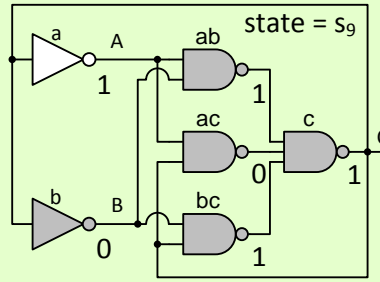

with new semi-modular definition

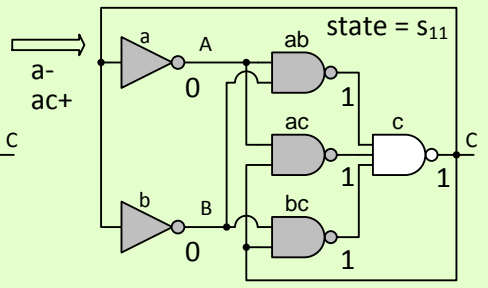

Fig. 3 Event trace in the new execution model. Events from $s_{0}$ to state $s_{8}$ are allowed under the old and new definitions of semi-modularity. With the old definition, the trace cannot exit $s_{8}$ without violating relative timing or semimodularity - the red box shows the forbidden exit with the semi-modularity violation. With the new definition, there is no semi-modularity violation-the trace proceeds correctly from s8 to state $s_{11}$-as shown in the green box.
To examine if the circuit with the relative timing constraints behaves as specified, we modeled the design set-up of Fig. 2 in NuSMV [12], using the standard interleaving model and both the old and the new definition of semi-modularity. The model checker reports a semi-modularity conflict when we use the old definition, Definition 1 , but it reports that the design behaves as specified when we use the new version, Definition 2. Fig. 3 shows the states and event trace leading up to the semi-modularity conflict under the old definition:

$$
s_{0} \stackrel{a+}{\longrightarrow} \stackrel{b+}{\longrightarrow} \stackrel{a b-}{\longrightarrow} s_{3} \stackrel{c+}{\longrightarrow} s_{4} \stackrel{b c-}{\longrightarrow} s_{5} \stackrel{b-}{\longrightarrow} s_{6} \stackrel{a b+}{\longrightarrow} s_{7} \stackrel{b c+}{\longrightarrow} s_{8}
$$

The transitions from initial state $s_{0}$ to state $s_{8}$ are allowed under both the old and the new definition of semi-modularity. All four relative timing constraints kick in at state $s_{4}$, after POD event $c+$, and start blocking the LATE events, $a$ - and $b$ - and $c$-, until the constraints are released by the corresponding EARLY events, $a c$ - or $b c$ - or both. Event $b$ - is released first, in state $s_{5}$. The other two remain blocked up to and including $s_{8}$.

Under the old definition, the trace stops at $s_{8}$ - execution cannot exit $s_{8}$, because all possible exits would violate either a relative timing constraint or semi-modularity. The forbidden exit causing the semi-modularity conflict is shown in the red box in the picture: event $a c$-causes the unstable gate $c$ to see its boolean input function $\neg(a b \wedge a c \wedge b c)$ change from 1 to 0 , before its output changes, i.e. before $c$-.

The new definition sees no semi-modularity conflict: $c$ - is not enabled in $s_{8}$, because it is blocked by a relative timing constraint, as indicated by the red stripes $(\backslash)$ for gate $c$. Under the new definition, execution proceeds correctly from $s_{8}$ to state $s_{11}$, and from there back to the initial state.

So, the execution model with Definition 1 finds the circuit with the four relative timing constraints incorrect. With Definition 2, it finds that the combination behaves as specified. In other words, the four constraints are rejected under Definition 1 and accepted under Definition 2.

Conclusion: Semi-modularity was-and is—an important paradigm in designing self-timed circuits that behave correctly independent of the gate and wire delays in the circuit. New design trends for fast and energyefficient self-timed circuits have increased the role of relative timing to fine-tune the circuits to better performance levels. But the event orderings expressed by relative timing interfere with the orderings imposed by "old school" semi-modularity. The paper solves this by providing a new definition of semi-modularity that fits rather than fights relative timing.

Hoon Park, Marly Roncken, and Xiaoyu Song (Asynchronous Research Center \& ECE Department, Portland State University, USA)

Anping He (School of Information Science and Engineering, Lanzhou University, China)

E-mail: hoon@cecs.pdx.edu

\section{References}

1 R. Miller, Switching Theory Volume 2: Sequential Circuits and Machines, Chapters 9-10. John Wiley \& Sons, 1965.

2 Victor Varshavsky (Ed.), Self-Timed Control of Concurrent Processes. Kluwer Academic Publ., 1990.

3 T. Meng, Synchronization Design for Digital Systems. Kluwer Academic Publ., 1991.

4 L. Lavagno and A. Sangiovanni-Vincentelli, Algorithms for Synthesis and Testing of Asynchronous Circuits. Kluwer Academic Publ., 1993.

5 R. Negulescu, "Process Spaces and Formal Verification of Asynchronous Circuits," PhD thesis, U. Waterloo, Canada, 1998.

6 K. Stevens, R. Ginosar, and S. Rotem, "Relative timing," in Proc. Adv. Research in Asynchronous Circuits and Systems, 1999, pp. 208-218.

7 J. Cortadella, M. Kishinevsky, A. Kondratyev, L. Lavagno, and A. Yakovlev, Logic Synthesis of Asynchronous Controllers and Interfaces. Springer-Verlag, 2002.

8 Y. Xu, "Algorithms for Automatic Generation of Relative Timing Constraints," PhD thesis, U. Utah, USA, 2011.

9 K. Desai, K. S. Stevens, and J. O'Leary, "Symbolic Verification of Timed Asynchronous Hardware Protocols," in Proc. IEEE Computer Society Annual Symposium on VLSI (ISVLSI), 2013, pp. 147-152.

10 J. Spars $\emptyset$ and S. Furber (Eds.), Principles of Asynchronous Circuit Design: A Systems Perspective. Kluwer Academic Publ., 2001.

11 A. Martin, "The Limitations to Delay-Insensitivity in Asynchronous Circuits," in Proc. Advanced Research in VLSI, 1990, pp. 263-278.

12 R. Cavada, A. Cimatti, C. Jochim, G. Keighren, E. Olivetti, M. Pistore, M. Roveri, and A. Tchaltsev, "NuSMV 2.4," 2013, http://nusmv.fbk.eu/. 